\title{
Spectrum Sensing for Cognitive Vehicular Networks over Composite Fading
}

\author{
Haroon Rasheed ${ }^{1}$ and Nandana Rajatheva ${ }^{1,2}$ \\ ${ }^{1}$ Telecommunications, Pathumthani 12120, SET, Asian Institute of Technology, Thailand \\ ${ }^{2}$ Center for Wireless Communications, University of Oulu, 90570 Oulu, Finland \\ Correspondence should be addressed to Haroon Rasheed, st105893@ait.ac.th
}

Received 16 August 2010; Revised 29 December 2010; Accepted 8 January 2011

Academic Editor: Cristina Pinotti

Copyright (c) $2011 \mathrm{H}$. Rasheed and N. Rajatheva. This is an open access article distributed under the Creative Commons Attribution License, which permits unrestricted use, distribution, and reproduction in any medium, provided the original work is properly cited.

\begin{abstract}
Recent advancement in vehicular wireless applications is also a major contributing factor in spectrum scarcity. Cognitive radio system is a mechanism which allows unlicensed cognitive users (CUs) to utilize idle unused bands. Fast and reliable detection of primary legacy user is the key component of cognitive radio networks. However, hidden terminal and low SNR problems due to shadow fading put fundamental limit to the sensing performance and practical entailments in design of the cognitive vehicular networks. Extensive modeling is being carried out to specify varying channel characteristics, particularly multipath fading and shadowing. Energy detection-(ED-) based spectrum sensing is a viable choice for many vehicle-to-vehicle (V2V) and vehicle to-road-side infrastructure (V2I) communications. This paper examines the performance of spectrum sensing using ED over Gamma-shadowed Nakagami-m composite fading channel to cater for both small-and-large scale fading. The results highlight the notable impact of shadowing spread and fading severity on detection performance. The relevant simulation results are presented to support our analytical results for average detection probability. Furthermore, these results are investigated and compared to other compound and classical channels.
\end{abstract}

\section{Introduction}

Radio spectrum is a limited resource, and almost all frequency bands are allocated to licensed users. The underutilization of spectrum bands extends to the definition of term spectrum hole as shown in Figure 1. It is actually an allotted band of frequencies, but at a specific time and geographic perspective, the band is not being employed by that user [1]. Cognitive radio is a compelling and innovative need for future wireless demands. There is a tremendous possibility to improve spectrum efficiency and quality of services through shared utilization. This system has also attracted a lot of interest in intelligent transportation networks (ITS). Cognitive radio-based vehicular transportation system in V2I and V2V communications and even interactions among on board devices within vehicles will help improve radio resource, energy efficiency, traffic network management, vehicular diagnostics and reduce accidents by road traffic awareness and route planning [2].
Spectrum sensing is the first step, as it should be carried out before permitting a cognitive client to approach an authorized channel. Fast and reliable detection of licensed user is the key component of cognitive radio networks. Thus, CUs need to have such cognitive abilities along with monitoring of substitute spectral route for possible band evacuation and minimum interference aggregation to other CUs [1].

In wireless communication, fading effect degrades signal propagation. This also places a primary constraint on sensing performance. Many propagation campaigns have been performed to investigate the statistics of shadow-fading in radio environments. Vehicle-to-roadside communications such as an automatic toll collection system has to grapple several channel variations particularly shadowing and multipath fading. Although the vehicle-mounted antenna ensures lineof-sight (LOS) connection to the beacon antenna, the shadow-fading due to reflections and scattering from the vehicle's own motor-hood or from other nearby large vehicles is a common problem as presented in Figure 2. 


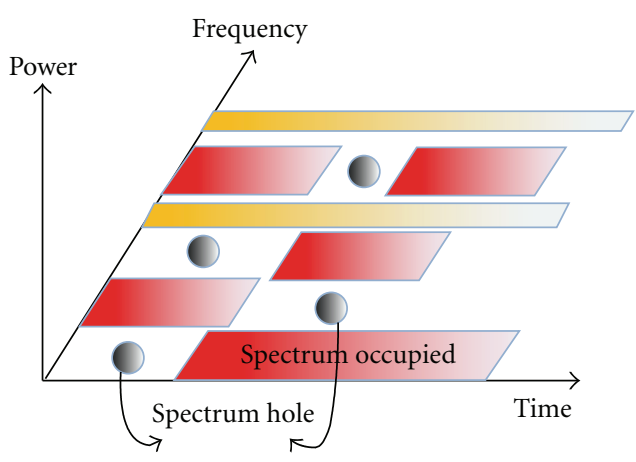

Figure 1: Spectrum holes concept.

Composite fading models like Suzuki, Loo, Rice-lognormal and Nakagami-lognormal, and so forth, are used to represent combine multipath and shadowing effects in wireless communications [3, pages 72-74]. They all rely on conventional lognormal distribution to model shadowing. However, capacity measurement and performance of spectrum sensing and access under multipath and shadowing using lognormal fading channel are somehow complex and do not lead to closed-form solutions. Alternatively, Gamma distribution is proposed to model the variations of average power which interprets shadow fading as close as lognormal distribution [4].

Energy detection [5] is proposed for spectrum sensing in cognitive radios due to its simplicity, low computational cost and ability to be applied on any kind of deterministic signal. In cognitive vehicular ad hoc network (VANET), ED can be applied to increase bandwidth efficiency. Licensed TV bands holders are primary users whereas vehicles and roadside infrastructure on highways and suburban cities will act as secondary users. As most of TV bands are blank, CUs in vehicular communication will perform ED-based sensing to find many unused spectrum bands. This assumption provides opportunistic spectrum access for wireless V2V and V2I communications. These ED-based cognitive vehicular systems lead to increased safety and information agility for vehicles on the road affected by multipath fading and shadowing. Moreover, the primary user transmission is modeled as a signal with known power, and hence energy detector is optimal [6, page 141]. In addition to cognitive radio, $\mathrm{ED}$ has found many applications in ultra wide-band technologies. Thus, performance analysis of energy detector in vehicular wireless networks with a variety of integrated techniques, is of particular interest.

The vehicular environment vision requires reliable, low latency wireless communication methods. One core issue is to detect and understand the nature of the wireless channel encountered by vehicular radios. The fading channels considered for are classical lognormal and Rayleigh fading channels. Previous research has primarily focused and examined ED, without (explicitly) taking into account fading channels that consist of composite distributions. The investigation over Gamma-shadowed Nakagami-m channel in $[7,8]$ consisted mainly on error performance, whereas spectrum sensing or estimation of detection probability is still an unexplored region.

This paper specifically focuses the performance analysis of energy detector in vehicular networks under Gammashadowed Nakagami-m fading channel model. The organization of the paper is as follows. In Section 2, detailed model of ED for spectrum sensing is discussed with its significance in vehicular networks. Section 3 defines the statistics of composite fading model with Gamma PDF as an alternative replacement for lognormal distribution. Composite fading channel statistics and signal-to-noise ratio (SNR) analysis for ED with respect to shadow-fading parameters are characterized in Section 4. The approximated expression for average detection probability over composite fading is obtained in Section 5 using series representation of Marcum-Q function. Further, Section 6 contains numerical and simulation results which also verify our theoretical formulations, and discussion about sensing and receiver performance in terms of fading parameters. Finally, Section 7 contains some concluding remarks.

\section{Energy Detection in Vehicular Technologies}

Energy detection is an efficient and fast noncoherent technique that essentially computes a running average of the signal power over a window of prespecified spectrum length. This is the simplest sensing method that requires no a priori knowledge about the transmitted signals. In addition to vehicular communications, the significance of energy detector finds many applications in wide-band technologies [9]. Performance analysis of ED over a variety of fading channels in vehicular network is considerably important and requires detailed investigation.

ED has already been recognized as an efficient sensing method for cognitive radio. In vehicular networks cognitive radio is a new paradigm to alleviate the bandwidth scarcity problem which will be an issue in near future. Similar to many cognitive radio systems, cognitive VANET faces the challenge of spectrum sensing, that is, the vehicles need to detect the presence or absence of licensed primary users with high reliability [9].

The block diagram of the energy detector is shown in Figure 3. The signal $x(t)$ is received and filtered with a bandpass filter (BPF) in order to limit the noise and to select the bandwidth of interest. The noise in the output of the filter has a band-limited, flat spectral density. Next, the energy detector consists a squaring device and a finite time integrator. The output signal $Y$ from the integrator as given in [5]

$$
Y=\frac{1}{T} \int_{t-T}^{t}|x(\tau)|^{2} d \tau .
$$

Finally, this output signal $Y$ is compared to the threshold $\lambda$, in order to decide whether a signal is present or not. The threshold is set according to the statistical properties of the output $Y$ when only noise is present. Thus, spectrum sensing is equivalent to detect the presence of an unknown 


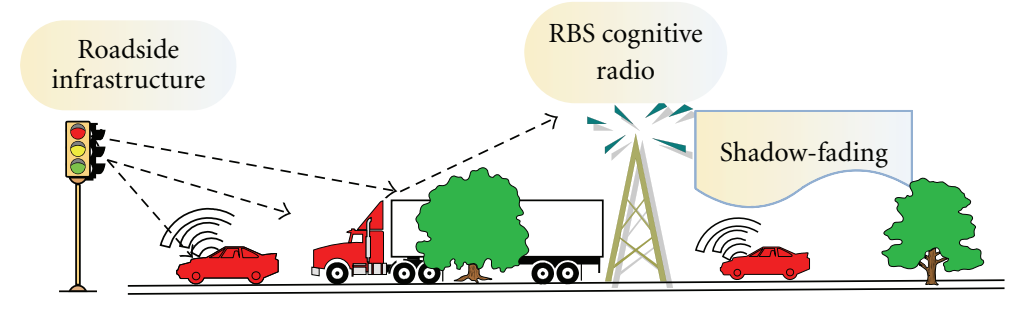

FIGURE 2: Shadowing in spatial environment.

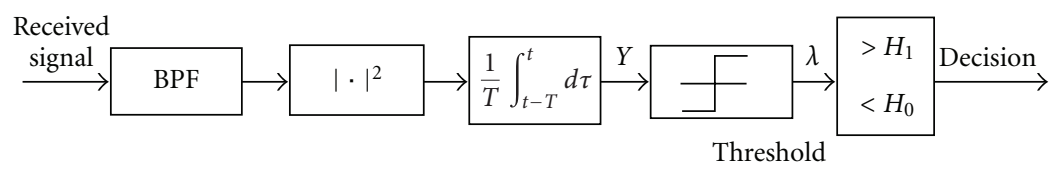

Figure 3: Block diagram of energy detector.

deterministic signal in the radio spectrum band which generally defines a binary hypothesis-testing problem as

$$
y(t)= \begin{cases}n(t) & H_{0}: \text { signal is absent, } \\ h x(t)+n(t) & H_{1}: \text { signal is present, }\end{cases}
$$

where $h$ is the channel response and $n(t)$ is the Gaussian random variable with zero mean and $N_{01} W$ variance; $n \sim$ $N\left(0, N_{01} W\right)$ where $N_{01}$ is the single sided noise spectral density and $W$ is the bandwidth. A sample function of time period $T$ of a process which is band limited to $W$ can be approximately described by a set of $2 u$ number of sample values, that is, $u=T W$, where $T$ and $W$ are selected to limit $u$ to an integer value [5]. Using this fact, the statistics of the detector decision variable $Y$ of the primary signal is a sum of $2 u$ zero and non zero mean Gaussian random variables under $H_{0}$ and $H_{1}$ respectively. The approximated noise energy over a time period $(0, T)$ for detector $Y$ is formulated as [10]

$$
Y=\frac{2}{N_{01}} \int_{0}^{T} n^{2}(t) d t=\sum_{i=1}^{2 u}\left(\frac{n_{i}}{\sqrt{N_{01} W}}\right)^{2}: H_{0}
$$

Similarly, $Y$ under $H_{1}$ is constructed as

$$
Y=\frac{2}{N_{01}} \int_{0}^{T} y^{2}(t) d t=\sum_{i=1}^{2 u}\left(\frac{h_{i} x_{i}+n_{i}}{\sqrt{N_{01} W}}\right)^{2}: H_{1} .
$$

Here, we assume that the $h$ is constant over the $2 u$ samples.

Thus, the probability density function (PDF) of $Y$ is a central chi-square variable $\chi_{2 u}^{2}$ with $2 u$ degrees of freedom for $H_{0}$ and noncentral chi-square variable $\chi_{2 u}^{2}(2 \gamma)$ with $2 u$ degrees of freedom and a noncentrality parameter $2 \gamma$ under $H_{1}$ respectively, which can be written as

$$
f_{Y}(y)=\left\{\begin{array}{l}
\frac{1}{2^{u} \Gamma(u)} y^{u-1} e^{-y / 2}: H_{0}, \\
\frac{1}{2}\left(\frac{y}{2 \gamma}\right)^{(u-1) / 2} e^{-(2 \gamma+y) / 2} I_{u-1}(\sqrt{2 \gamma y}): H_{1},
\end{array}\right.
$$

where $\gamma$ is the SNR, that is, $\gamma=h^{2} E_{s} / N_{01}$ and $E_{s}$ is defined as observed signal energy, $\Gamma(\cdot)$ is gamma function and $I_{n}(\cdot)$ is $n$th order modified Bessel function of the first kind. The exact closed-form equations for probabilities of detection $P_{d}$, false alarm $P_{f}$, and missed detection $P_{m}$ over additive white Gaussian noise (AWGN) channel are given by [10]

$$
P_{d}=P_{r}\left\{Y>\lambda \mid H_{1}\right\}=Q_{u}(\sqrt{2 \gamma}, \sqrt{\lambda}),
$$

where $\lambda$ is the threshold for signal sample and $Q_{M}(\cdot, \cdot)$ is generalized $M$ th order Marcum-Q function defined as [11, Equation (2.1-122), page 44]

$$
Q_{M}(\alpha, \beta)=\int_{\beta}^{\infty} \frac{t^{M}}{\alpha^{M-1}} e^{-\left(t^{2}+\alpha^{2}\right) / 2} I_{M-1}(\alpha t) d t,
$$

where $I_{M-1}(\cdot)$ is the modified Bessel function of $(M-1)$ th order. The probability of false alarm is expressed as

$$
P_{f}=P_{r}\left\{Y>\lambda \mid H_{0}\right\}=\frac{\Gamma(u, \lambda / 2)}{\Gamma(u)},
$$

where $\Gamma(\cdot, \cdot)$ is an upper incomplete gamma function which is defined as $\Gamma(m, n)=\int_{n}^{\infty} t^{m-1} e^{-t} d t$ [12, Equation (8.350-2), page 899]. Since $\gamma$ does not appear in (8), average false alarm probability over any fading channel will be similar (8).

Threshold $\lambda$ for ED is calculated for a specified $P_{f}$ using (8), whereas conventional optimality principle, NeymanPearson criterion maximizes $P_{d}$ for a given $P_{f}$ and equivalent to the likelihood ratio test (LRT) of $Y$ indicated as $\operatorname{LR}(Y)$. In general, there is no LOS path present between the secondary user and the primary transmitter. Hence, the received primary signal is a superposition of many non LOS signals and is well approximated to Gaussian random variables according to central limit theorem [13]. In our consideration, when both the primary signal and noise are treated as Gaussian processes, energy detector can meet any desired $P_{d}$ and $P_{f}$ simultaneously, hence the threshold $\lambda$ is optimal [14]. Finally, the probability of missed detection can be evaluated as

$$
P_{m}=1-P_{r}\left\{Y>\lambda \mid H_{1}\right\}=1-Q_{u}(\sqrt{2 \gamma}, \sqrt{\lambda}) .
$$


Since $P_{f}$ is independent of SNR due to the concept of no signal transmission, $P_{d}$ in (6) is dealt only for nonfading environment, where channel $h$ is deterministic. While a varying $h$ due to shadowing and multipath fading gives probability of detection on the instantaneous SNR $\gamma$ [15]. In this case, the average probability of detection $P_{d}$ is assessed by averaging (6) over fading statistics.

The IEEE $802.22 \mathrm{draft}$ standard addresses fixed-access devices and targets rural area applications. Energy detector has been tested for spectrum sensing in cognitive radio. Also ED is a non coherent reception technique which is implementable either using analog or digital design. The experimental test bed description and algorithms of ED in cognitive radio are given in $[16,17]$. The SNR limitation is dealt with the help of collaboration of users [18] and continuous research work is ongoing to overcome ED limitations.

\section{Composite Channel Model for Shadow Fading}

In vehicular communication, a signal is assumed to pass through a large number of attenuating materials before reaching to the destination. Owing to the short range of typical V2V and V2I links, a more precise description of the fluctuations is often given by small-scale fading models. These fluctuations are caused by constructive and destructive interference between multipath components. Nakagami distribution can model fading conditions like Rayleigh or Rician statistics, depending on whether or not there is a LOS. The fading statistics for V2V propagation channel under realistic suburban driving conditions is modeled and analyzed by Nakagami distribution [19].

The PDF $f_{X}(x)$ of the envelope $X$, under Nakagami fading conditions describes the magnitude of the received envelope by the distributions given as

$$
f_{X}(x)=\frac{2 m^{m} x^{2 m-1} e^{-(m / p) x^{2}}}{\Gamma(m) p^{m}}, \quad x \geq 0.5,
$$

where $\Gamma(\cdot)$ is the gamma function and $m$ is the Nakagami fading parameter. $p=E\left[x^{2}\right]$ is the average power of the received signal, $E[\cdot]$ represents statistical average operator.

The average power $p$ is deterministic in the absence of shadowing. However, V2I and V2V channel variations due to obstructions and large size vehicles, and so forth, exhibit shadowing effect. Thus, mean power of the signal becomes random and (10) can be written by conditioning the envelope as [20]

$$
f_{X \mid P}(x \mid p)=\frac{2 m^{m} x^{2 m-1} e^{-(m / p) x^{2}}}{\Gamma(m) p^{m}}, \quad x, p>0 .
$$

The composite PDF with fading and shadowing is therefore written as

$$
f_{X}(x)=\int_{0}^{\infty} f_{X \mid P}(x \mid p) f_{P}(p) d p,
$$

where $f_{P}(p)$ is the PDF of average power due to shadowing. If $f_{P}(p)$ is lognormally distributed and $m \neq 1, f_{X}(x)$ will be
Nakagami-lognormal composite distribution. Nevertheless, due to the inherent analytical complication of handling correlated lognormal random variables, it is often inconvenient for further performance measurements and will not lead to a closed-form solution $[15,20]$.

3.1. Gamma Distribution: Alternative to Lognormal Distribution. Lognormal distribution is generally used to model average power variations. Empirical studies have shown that $p$ has a lognormal PDF [21, Equation (1.5), page 21], that is,

$$
f_{P}(p)=\frac{1}{p \sqrt{2 \pi \sigma^{2}}} \exp \left(-\frac{\left(20 \log p-\mu_{\mathrm{dBm}}\right)^{2}}{2 \sigma^{2}}\right), \quad p>0,
$$

where $\sigma$ is the standard deviation for shadowing, whereas $\sigma \rightarrow 0$ corresponds to no shadowing. The local mean power $p$ fluctuates about a constant area mean power $\mu_{\mathrm{dBm}}$, that is, $\mu_{\mathrm{dBm}}=30+10 E\left[\log _{10} p\right]$ [21, Equation (2.202), page 98]. Composite fading channel with Nakagami PDF and lognormally distributed power has involved complicated integral form, also from above discussion even fitting this PDF to real data in vehicular communications is a difficult task. Hence, a closed-form expression for various system computations is a challenging problem. Based on theoretical results and measured data, an alternative substitute is the two-parameter Gamma distribution, which approximates several PDFs and justifies the lognormal distribution [4, 22].

Lognormal and Gamma PDFs are interchangeable in simulating real data when $\sigma_{\mathrm{dB}}$ is not large, that is, $\leq 6$ [23]. For this, we compared the average detection probability variations over Gamma and lognormal fading channels in Figure 4 for Gamma shadowing parameter $m_{0}$ with respective lognormal $\sigma_{\mathrm{dB}}$ spreads. It is evident from simulation results that for $\sigma_{\mathrm{dB}}<6$, Gamma PDF is a good fit for lognormal distribution. Furthermore, when signal amplitude follows Nakagami-m distribution, the PDF of SNR $\gamma$, which is the sum of $n$ independent and identically distributed (i.i.d) exponential random variables is Gamma distributed.

Considering $P=X^{2}$, the obtained result will be the PDF of $P$ can be expressed as [11, Equation (2.1-105), page 41]

$$
f_{P}(p)=\frac{1}{\sqrt{2 \pi \sigma^{2} p}} \exp \left(-\frac{p}{2 \sigma^{2}}\right), \quad p \geq 0
$$

if $P$ is defined as $P=\sum_{i=1}^{n} X_{i}^{2}$, where $i=1,2, \ldots, n$ are statistically i.i.d Gaussian random variables with zero mean and variance $\sigma^{2}$. The characteristics function of $P$ is represented as [11, Equation (2.1-107), page 41]

$$
\phi_{P}(j v)=\frac{1}{\left(1-j 2 v \sigma^{2}\right)^{n / 2}} .
$$

The inverse transform of this characteristic function leads to central chi-square distribution with $n$ degree of freedom, that is,

$$
f_{P}(p)=\frac{p^{(n / 2)-1}}{\sigma^{n} 2^{n / 2} \Gamma(n / 2)} \exp \left(-\frac{p}{2 \sigma^{2}}\right), \quad p \geq 0
$$




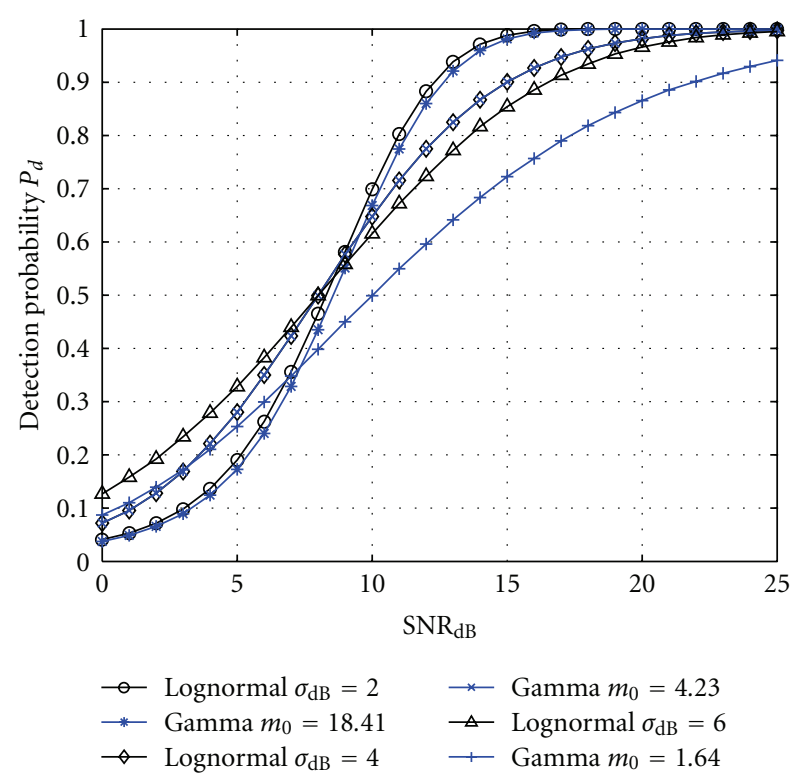

Figure 4: A comparison of average detection probability $\left(P_{d}\right)$ variations between lognormal $\sigma_{\mathrm{dB}}$ spread and Gamma distributions shadowing parameter $m_{0}$ values, keeping time bandwidth product $u=5$ and false alarm probability $P_{f}=0.01$.

TABLE 1: Gamma $m_{0}$ and lognormal $\sigma_{\mathrm{dB}}$ values relationships.

\begin{tabular}{cccc}
\hline$\sigma_{\mathrm{dB}}$ & 2 & 4 & 6 \\
\hline$m_{0}$ & 18.41 & 4.23 & 1.64 \\
\hline
\end{tabular}

substituting $p_{0}=2 \sigma^{2}$ and $m_{0}=n / 2$ is simplified to Gamma PDF as

$$
f_{P}(p)=\frac{p^{m_{0}-1}}{\Gamma\left(m_{0}\right) p_{0}^{m_{0}}} \exp \left(-\frac{p}{p_{0}}\right)
$$

where $p_{0}$ is the measure of average power and $m_{0}$ is the order of Gamma PDF that inversely reflects shadowing severity. By changing $m_{0}$, several distributions like lognormal, Gaussian, and so forth, can be obtained $[8,20]$. The parameters $m_{0}$ and $p_{0}$ of Gamma distribution are related with their respective lognormal mean and the variance as $m_{0}=1 /\left(e^{\sigma^{2}}-1\right)$ and $p_{0}=\mu \sqrt{\left(m_{0}+1\right) / m_{0}}$, where $\sigma=\sigma_{\mathrm{dB}} / 8.686$ and $\mu$ are defined as the standard deviation and average power related to lognormal PDF respectively. The equivalent values of $\sigma_{\mathrm{dB}}$ and $m_{0}$ are found accordingly and indicated in Table 1.

\subsection{Gamma-Shadowed Nakagami-m Fading Distribution for} Multipath and Shadowing. In radio propagation, shadowing and multipath fading appear simultaneously. Conversely, the channel model most frequently employed in vehicular communication do not distinguish the two effects. The Gamma-shadowed Nakagami-m (composite) distribution provides analytical restraint in terms of simplicity with which parameters can be computed. By substituting (17) and (11)

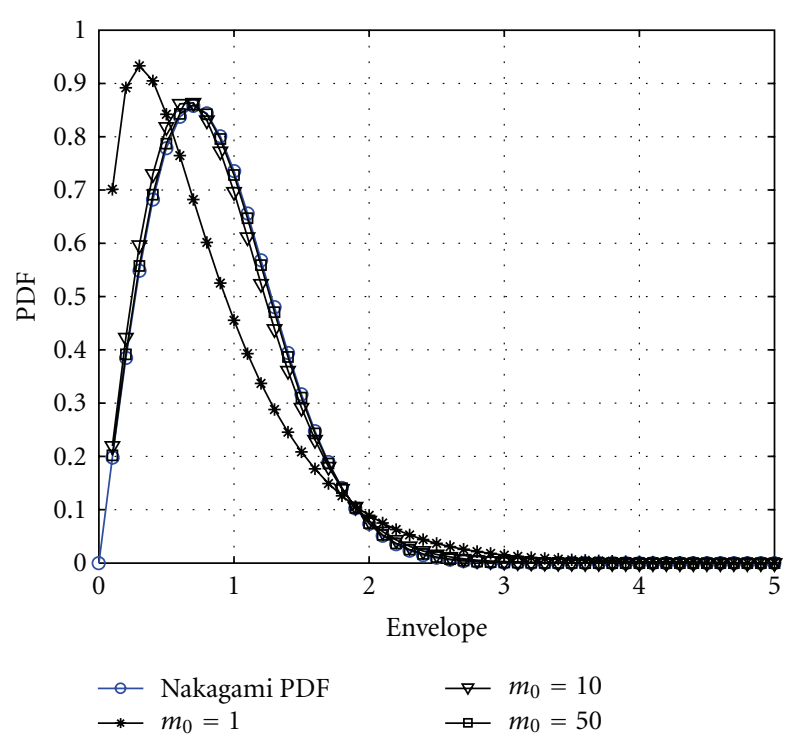

FIGURE 5: The composite PDF over various shadowing parameter $m_{0}$ values with corresponding Nakagami-m keeping $m=0.8$.

in (12), the compound envelope of $X$ consists of both multipath and shadowing is obtained in (18) as [20]

$$
\begin{aligned}
f_{X}(x) & =\int_{0}^{\infty} f_{X \mid P}(x \mid p) f_{P}(p) d p \\
& =\frac{2 c}{\Gamma\left(m_{0}\right) \Gamma(m)}\left(\frac{c x}{2}\right)^{m_{0}+m-1} K_{m_{0}-m}(c x), \quad x>0,
\end{aligned}
$$

where $c=2 \sqrt{m / p_{0}}$ and $K_{m_{0}-m}()$ is the modified Bessel function of order $\left(m_{0}-m\right)$.

The numerically evaluated PDFs of composite and Nakagami fading are plotted in Figure 5. It is found that when the effect of shadowing is decreased at large value of $m_{0}=50$, the composite PDF exactly overlaps the Nakagami PDF and represents both multipath and shadowing effects.

\section{Composite Fading Channel Statistics}

The moments of the compound envelope PDF are expressed as [20]

$$
E_{c}\left[X^{t}\right]=\frac{\Gamma\left(m_{0}+t / 2\right) \Gamma(m+t / 2)}{\Gamma\left(m_{0}\right) \Gamma(m)}\left(\frac{2}{c}\right)^{t} .
$$

From above formula, amount of fading (AF) defined as $A_{f}$ can be obtained as [20]

$$
\begin{aligned}
A_{f} & =\frac{\text { variance }\left[X^{2}\right]}{E\left[X^{2}\right]^{2}} \\
& =\frac{m m_{0}+m^{2} m_{0}+m m_{0}^{2}}{m^{2} m_{0}^{2}}>0 .
\end{aligned}
$$

The value of $A_{f}$ ranges from zero to infinity, corresponding to no fading to severe multipath fading and shadowing. By 


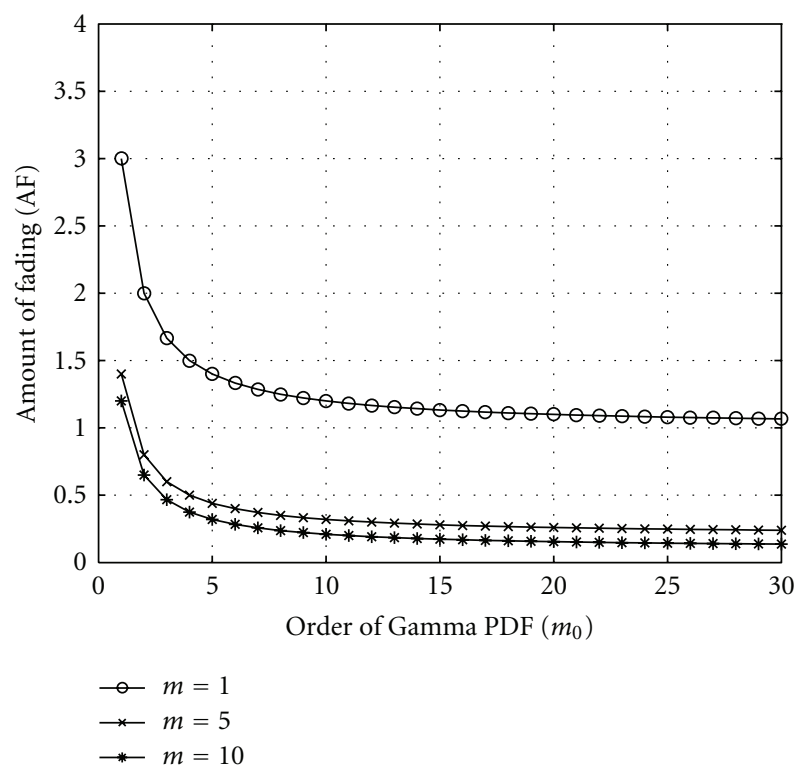

Figure 6: Amount of fading with respect to shadowing parameter $m_{0}$ taking different values of fading severity parameter $m$.

using (20) AF curves are simulated over a range of $m_{0}$ for various $m$ values as depicted in Figure 6. It is found that AF is related inversely with fading severity, and become independent for shadowing parameter $m_{0}>5$ corresponds to $3.71 \sigma_{\mathrm{dB}}$ spread.

For a given noise uncertainty, there exist a SNR wall, below which the ED cannot notice the existence of unknown deterministic signal [14]. Due to shadowing and fading effects, it is possible that user experiences very low SNR conditions and hence, its performance diminishes. For detection improvement in such situations, channel SNR values associated with the shadow-fading condition must be monitored carefully. The SNR of composite envelope is obtained from (19) as given by [20]:

$$
\mathrm{SNR}=\frac{1}{\sqrt{m_{0} m\left[\Gamma\left(m_{0}\right) \Gamma(m) / \Gamma\left(m_{0}+0.5\right) \Gamma(m+0.5)\right]^{2}-1}} .
$$

By using (21), we plotted SNR variations as a function of fading severity index $m$ depicted in Figure 7 . It is observed that larger values of $m_{0}$ correspond to high SNR values. However, for $m>0.5$ change in SNR gradually decreases. When the user is in shadow fading environment with low SNR, the detection threshold $\lambda$ of ED should be adjusted adaptively.

\section{Average Detection Probability over Composite Fading Channel}

The average probability of detection $\bar{P}_{d}$ over fading statistics is determined as [10]:

$$
\bar{P}_{d}=\int_{0}^{\infty} Q_{u}(\sqrt{2 \gamma}, \sqrt{\lambda}) f_{\gamma}(\gamma) d \gamma
$$

where $f_{\gamma}(\gamma)$ is the PDF of SNR under shadow fading.

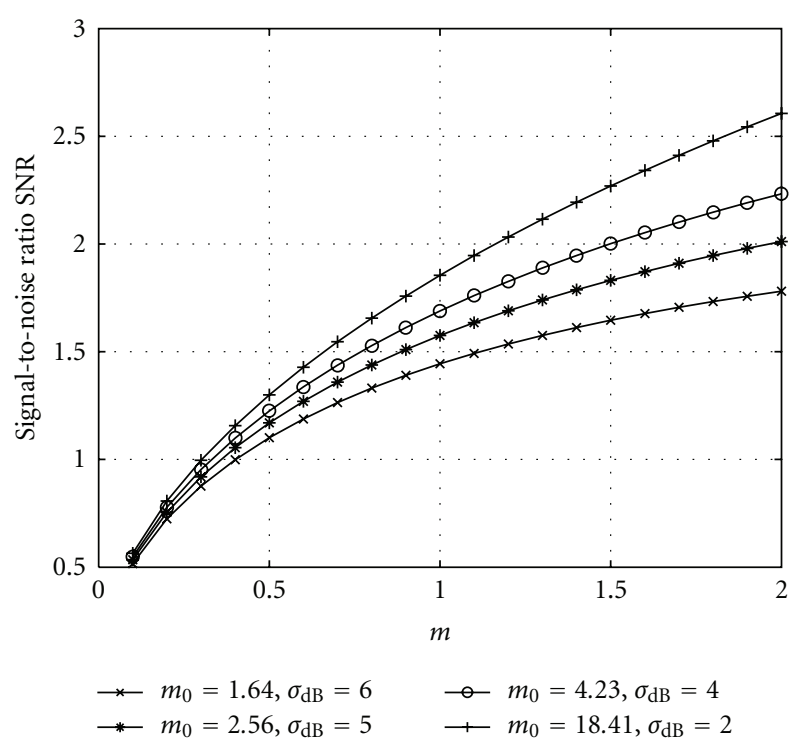

FIGURE 7: Composite fading channel signal-to-noise ratio (SNR) as a function of shadowing parameter $m_{0}$ over different values of fading severity parameter $m$.

When envelope of received signal due to shadow-fading is modeled as Nakagami distribution the SNR PDF can be modeled as Gamma distribution [20]

$$
f_{\gamma \mid \bar{\gamma}}(\gamma)=\frac{m^{m} \gamma^{m-1}}{\Gamma(m) \bar{\gamma}^{m}} \exp \left(-\frac{m \gamma}{\bar{\gamma}}\right), \quad \gamma \geq 0, m \geq 0.5,
$$

where $\Gamma(\cdot)$ is the gamma function and $m$ is the Nakagami fading parameter. $\bar{\gamma}=E\left[h^{2} E_{b} / N_{01}\right]$ is the average SNR or average power of the received signal being random, hence reflects the existence of shadowing. The two-parameter Gamma distribution which has shown a good justification of lognormal PDF is defined as

$$
f_{\bar{\gamma}_{0}}(\gamma)=\frac{m_{0}^{m_{0}} \gamma^{m_{0}-1}}{\Gamma\left(m_{0}\right) \bar{\gamma}_{0}^{m_{0}}} \exp \left(-\frac{\gamma}{\bar{\gamma}_{0}}\right),
$$

where $\bar{\gamma}_{0}$ is the measure of average power and is related to the average SNR $\bar{\gamma}, m_{0}$ is order of Gamma PDF and a measure of channel shadowing. The PDF of received SNR in combined shadow fading situation is given as [20]

$$
f_{\gamma}(\gamma)=\int_{0}^{\infty} f(\gamma \mid \bar{\gamma}) f_{P}(p)
$$

Substituting (23) and the two-parameter Gamma PDF from (24) in (25), and by changing variables $f_{\gamma}(\gamma)$, the PDF of received SNR in combined shadow fading environment can be obtained as [8]

$$
\begin{aligned}
f_{\gamma}(\gamma)= & \frac{2}{\Gamma(m) \Gamma\left(m_{0}\right)}\left(\frac{c_{0}}{2}\right)^{m_{0}+m} \\
& \times \gamma^{\left(\left(m_{0}+m\right) / 2\right)-1} K_{m_{0}-m}\left(c_{0} \sqrt{\gamma}\right), \quad \gamma>0,
\end{aligned}
$$

where $c_{0}=2 \sqrt{m m_{0} / \bar{\gamma}_{0}}$ is scaling parameter related to $\bar{\gamma}$ and $K_{m_{0}-m}(\cdot)$ is the modified Bessel function of order $\left(m_{0}-m\right)$. 
The probability of detection over fading environment is evaluated using complicated integral forms involving Marcum-Q function. Further, these integrals cannot be solved in closed-forms in general with the available integral results [24]. In order to avoid such mathematical difficulties and to evaluate integrals involving Marcum- $Q$ function with exponentials and Bessel functions having complicated arguments, alternative series representation of Marcum-Q function is used.

The generalized Marcum-Q function $Q_{u}(a, b)$ in its alternative series representation for $\sqrt{2 \gamma}>\sqrt{\lambda} \geq 0$, as given in $[24]$

$$
Q_{u}(\sqrt{2 \gamma}, \sqrt{\lambda})=1-e^{(-(2 \gamma+\lambda) / 2)} \sum_{n=u}^{\infty}\left(\frac{\lambda}{2 \gamma}\right)^{n / 2} I_{n}(\sqrt{2 \lambda \gamma}),
$$

where $I_{n}(\cdot)$ is the $n$th order modified Bessel function of the first kind. By substituting (27) and (26) in (22), the average detection probability over compound fading channel $P_{d}$ can be written as

$$
\begin{aligned}
\bar{P}_{d}= & \int_{0}^{\infty}\left(1-e^{(-(2 \gamma+\lambda) / 2)} \sum_{n=u}^{\infty}\left(\frac{\lambda}{2 \gamma}\right)^{n / 2} I_{n}(\sqrt{2 \lambda \gamma})\right) \\
& \times \frac{2}{\Gamma(m) \Gamma\left(m_{0}\right)}\left(\frac{c_{0}}{2}\right)^{m_{0}+m} \gamma^{\left(\left(m_{0}+m\right) / 2\right)-1} K_{m_{0}-m}\left(c_{0} \sqrt{\gamma}\right) d \gamma .
\end{aligned}
$$

By using the fact $\int_{0}^{\infty} f_{\gamma}(\gamma) d \gamma=1,(28)$ will be simplified as,

$$
\begin{aligned}
\bar{P}_{d}= & -\frac{2 e^{-\lambda / 2}}{\Gamma(m) \Gamma\left(m_{0}\right)}\left(\frac{c_{0}}{2}\right)^{m_{0}+m} \sum_{n=u}^{\infty}\left(\frac{\lambda}{2}\right)^{n / 2} \\
& \times \int_{0}^{\infty} e^{-\gamma} \gamma^{\left(-n+m_{0}+m\right) / 2-1} I_{n}(\sqrt{2 \lambda \gamma}) K_{m_{0}-m}\left(c_{0} \sqrt{\gamma}\right) d \gamma .
\end{aligned}
$$

However, integral of the Bessel functions product $I_{n}(\cdot)$ and $K_{m_{0}-m}(\cdot)$ with exponentials and powers does not lead to a closed-form, therefore we evaluated (29) numerically and compared it with our simulation results.

\section{Numerical and Simulation Results}

The performance of energy detector over composite fading channel for vehicular communications is presented in terms of average detection probability $\bar{P}_{d}$ and complementary receiver operating characteristic (ROC) curves, that is, $P_{m}$ versus $P_{f}$. By varying the average SNR $\bar{\gamma}_{0}$, while keeping all the other parameters such as threshold $\lambda$, time bandwidth product $u$, fading metric $m$ and shadowing parameter $m_{0}$ constant, the behavior of $\bar{P}_{d}$ is shown. In the second scenario, detector performance is evaluated by means of complementary ROC curves similar to [10], that is, $\bar{P}_{m}$ versus $P_{f}$ where $\bar{P}_{m}=1-\bar{P}_{d}$.

Detector behavior characterization over various parameters is important in order to adjust numerous vehicular environments. The detector threshold $\lambda$ is calculated for

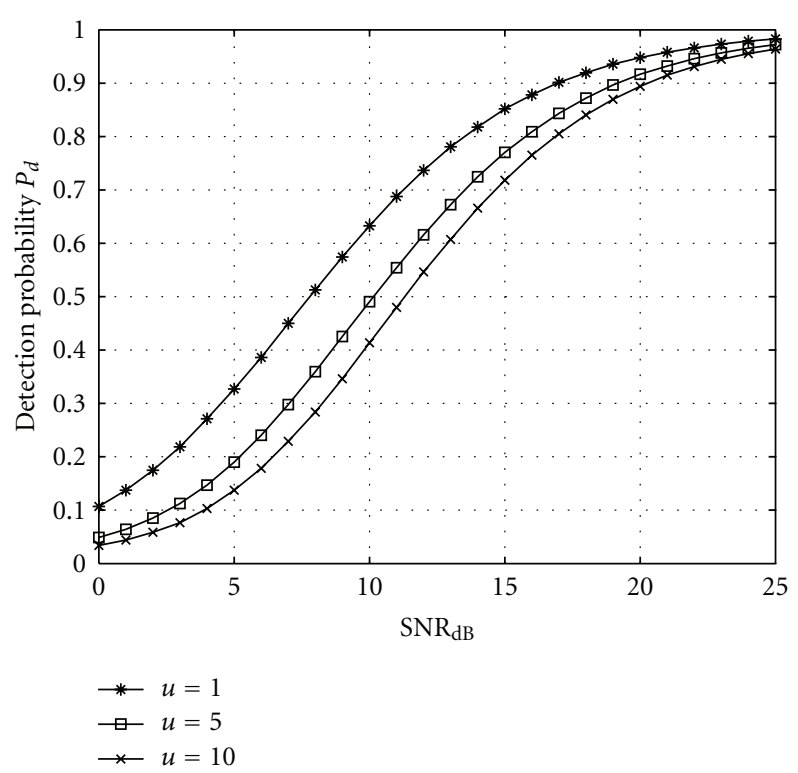

FIGURE 8: Average detection probability $\left(\bar{P}_{d}\right)$ variations over various time bandwidth product $(u)$ values in compound fading channel while taking false alarm probability $P_{f}=0.01$.

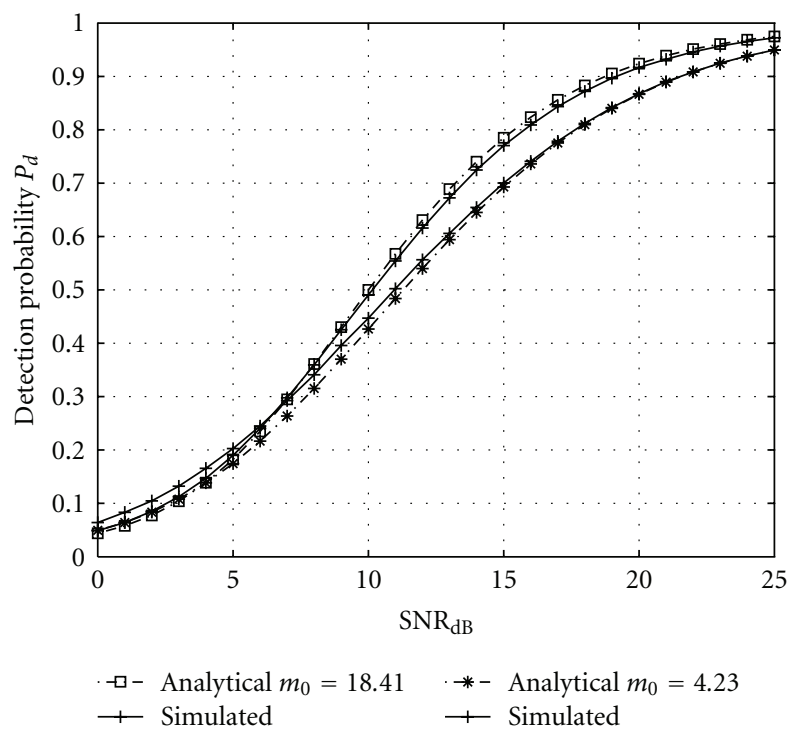

Figure 9: Average detection probability $\left(\bar{P}_{d}\right)$ both analytical and simulation for composite fading taking $m=1, u=5$, and $P_{f}=$ 0.01 .

$P_{f}=0.01$ from (8) taking $u=1,5,10$ as shown in Figure 8. It can be observed that the detector performance with smaller number of samples (lower $u$ ) for energy $E_{s}$ is better if compared with that obtained from greater number of samples. The detector threshold $\lambda$ is found at specified $P_{f}$. An increase in the threshold value of the detector, that is, larger $u$ leads to reduction in both the false alarm and detection probabilities.

By using (29) average $\bar{P}_{d}$ over varying $\bar{\gamma}_{0}$ is computed with the help of MATHEMATICA 7. Figure 9 illustrates 


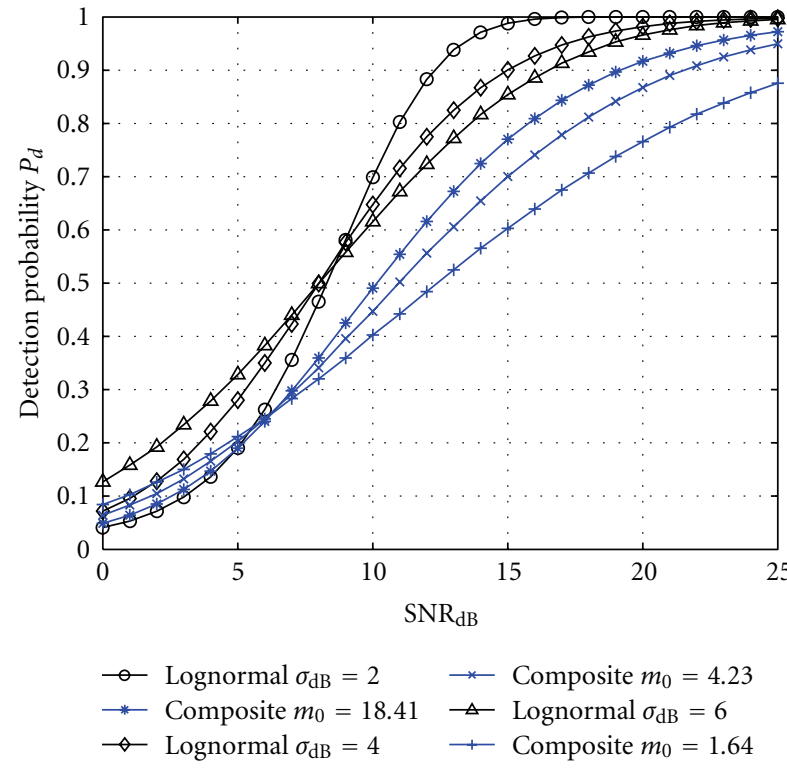

Figure 10: A comparison of average detection probability $\left(\bar{P}_{d}\right)$ variations between lognormal and composite fading channel keeping fading severity parameter $m=1$, time bandwidth product $u=5$ and false alarm probability $P_{f}=0.01$.

the average $P_{d}$ variations over composite fading channel at $m_{0}=18.41$ and 4.23 corresponding to $\sigma_{\mathrm{dB}}=2$ and 6 respectively. Both analytical and simulated results interpret sufficient detector performance over high average SNR, that is, SNR > $10 \mathrm{~dB}$, also higher values of $m_{0}$ reflects better detection over specified $\mathrm{SNR}_{\mathrm{dB}}$. However, for low $\mathrm{SNR}$ region the deteriorating performance can be improved using spatial diversity and appropriate combining techniques.

The detection performance of composite fading channel rendering both multipath and shadowing effects at different range of $m_{0}$ is evaluated in Figure 10. The better detection is illustrated as the effect of shadowing diminishes at higher values of $m_{0}$, keeping fading characteristics $m=1$ unchanged. The similar phenomenon is obtained at smaller values of lognormal shadowing statistics $\sigma_{\mathrm{dB}} \leq 2$ referring to light to moderate shadowed scenario. Whereas for heavier shadowed region, that is, $\sigma_{\mathrm{dB}}>6$ to achieve a good fit is not possible. However, fading severity index $m>1$ keeps composite channel to overcome the shadowing and LOS communication can be considered which is common in vehicular networks.

Complementary ROC plot $\left(P_{m}\right.$ versus $\left.P_{f}\right)$ for composite channel is plotted in contrast to Gamma, lognormal and Nakagami channels as shown in Figure 11. Both the Gamma and lognormal are offering similar detection characteristics for the selected parameters.It is evident that composite fading channel exhibits the combine fading properties of Gamma and Nakagami-m fading channel.

In Figure 12 the ROC curve of Gamma-shadowed Nakagami-m composite fading clearly indicates a substantial detection performance improvement in contrast to Loo and

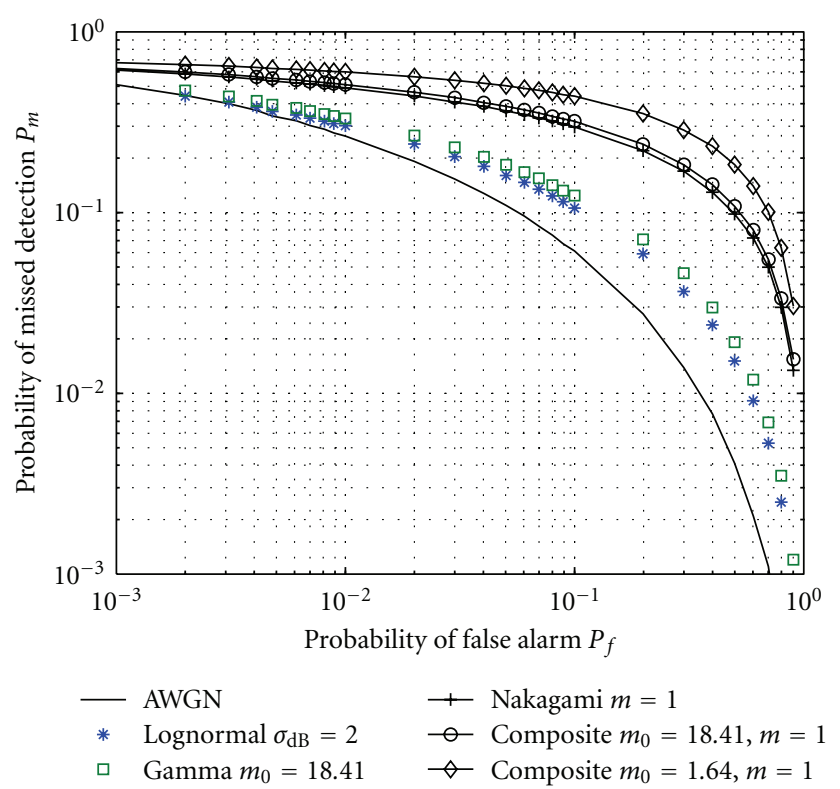

Figure 11: Complementary ROC $\left(P_{m}\right.$ versus $\left.P_{f}\right)$ of composite fading channel over different shadowing parameter $m_{0}$ values in comparison with Nakagami-m fading channel. AWGN curve is provided for reference.

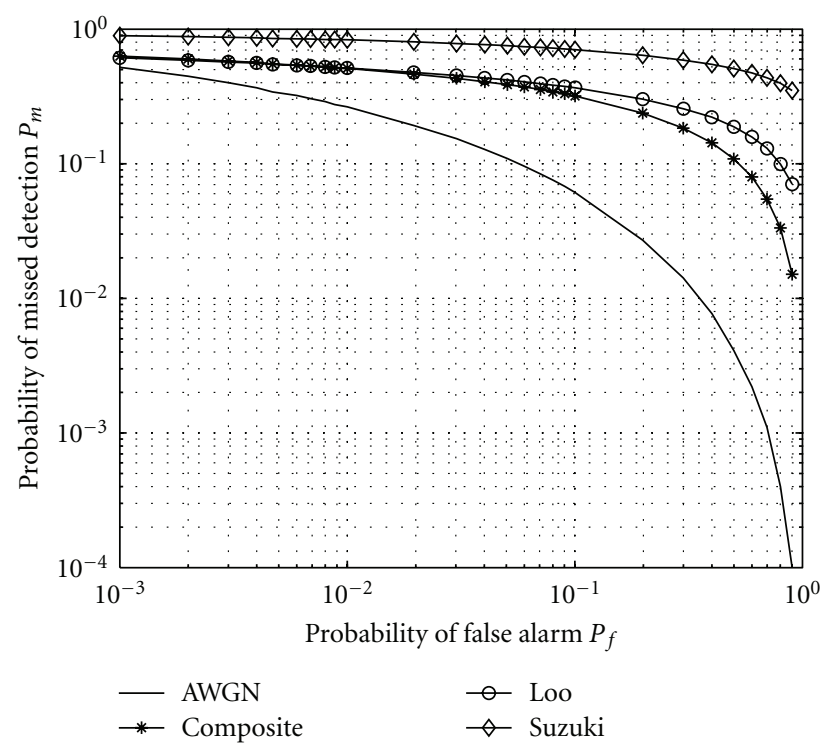

Figure 12: Complementary ROC $\left(P_{m}\right.$ versus $\left.P_{f}\right)$ of different mixed distribution fading channel over shadowing factor $\sigma_{\mathrm{dB}}=2$ and fading severity parameter $k=m=1$. AWGN curve is provided for reference.

Suzuki mixed distribution channels where $k$ represents carrier to multipath ratio in Loo distribution. Another prominent feature of the versatile Gamma-shadowed Nakagami$\mathrm{m}$ composite channel is having minimum set of parameters as summarized in Table 2 from which diverse shadow fading environments can be approximated. 
TABLE 2: Input parameters of different shadow fading distributions.

\begin{tabular}{lccccc}
\hline Channel/parameter & $m$ & $m_{0}$ & $\sigma_{\mathrm{dB}}$ & $k$ & $\mu_{\mathrm{dB}}$ \\
\hline Composite & 1 & 18.41 & - & - & - \\
Loo & - & - & 2 & 5 & -6 \\
Suzuki & - & - & 2 & - & -6 \\
\hline
\end{tabular}

\section{Conclusion}

In the standardization process of vehicular networks, channel models are required to evaluate and select the proposed physical layer modulation and coding schemes. We have presented spectrum sensing using energy detection over Gamma-shadowed Nakagami-m composite fading model. The scheme can be effectively deployed in vehicular networks and help to combat against spectrum scarcity. To avoid computational complexities of the integrals involving Marcum-Q function, we apply PDF-based approximation and alternative series representation of generalized Marcum-Q function. Employing these approaches the average detection probability is evaluated. Analytical and simulation results are provided to support the theoretical formulations and derivations. The presented results show that spectrum sensing and access in vehicular communication can be improved by modeling the wireless environment precisely. Gamma-Shadowed Nakagami-m fading channel based energy detection provides fast and reliable sensing in cognitive vehicular networks. The numerical and simulation results provide insight and can serve as a quick way of assessing performance. From the presented results it is clear that a channel model composed of mixed distributions is useful for designing vehicular wireless systems and networks.

\section{References}

[1] S. Haykin, "Cognitive radio: brain-empowered wireless communications," IEEE Journal on Selected Areas in Communications, vol. 23, no. 2, pp. 201-220, 2005.

[2] H. Hannes and P. L. Kenneth, Eds., VANET: Vehicular Applications and Inter-Networking Technologies, John Wiley \& Sons, New York, NY, USA, 2010.

[3] E. G. Corazza, Digital Satellite Communications, Springer, New York, NY, USA, 2007.

[4] A. Abdi and M. Kaveh, "On the utility of gamma PDF in modeling shadow fading (slow fading)," in Proceedings of the 49th IEEE Vehicular Technology Conference, vol. 3, pp. 23082312, May 1999.

[5] H. Urkowitz, "Energy detection of unknown deterministic signals," Proceedings of the IEEE, vol. 55, pp. 523-531, 1967.

[6] S. Kay, Fundamentals of Statistical Signal Processing: Detection Theory, Prentice-Hall, Englehood Cliffs, NJ, USA, 1998.

[7] P. M. Shankar, "Error rates in generalized shadowed fading channels," Wireless Personal Communications, vol. 28, no. 3, pp. 233-238, 2004.

[8] I. M. Kostic, "Analytical approach to performance analysis for channel subject to shadowing and fading," IEE Proceedings on Communications, vol. 152, no. 6, pp. 821-827, 2005.

[9] L. Catalin, K. V. Rama, A. Onur, B. Dusan, and S. Ivan, "Evaluation of energy-based spectrum sensing algorithm for vehicular networks," in Proceedings of the Software Defined
Radio and Dynamic Spectrum Access Technical Conference, Washington, DC, USA, December 2009.

[10] F. F. Digham, M. S. Alouini, and M. K. Simon, "On the energy detection of unknown signals over fading channels," IEEE Transactions on Communications, vol. 55, no. 1, pp. 21-24, 2007.

[11] J. G. Proakis and M. Salehi, Digital Communications, McGraw Hill, New York, NY, USA, 5th edition, 2008.

[12] S. I. Gradshteyn and M. I. Ryzhik, Table of Integrals, Series, and Products, Academic Press, 7th edition, 2007.

[13] J. Ma, G. Zhao, and Y. Li, "Soft combination and detection for cooperative spectrum sensing in cognitive radio networks," IEEE Transactions on Wireless Communications, vol. 7, no. 11, pp. 4502-4507, 2008.

[14] A. Sahai, N. Hoven, and R. Tandra, "Some fundamental limits on cognitive radio," in Proceedings of the 42nd Allerton Conference on Communication, Control, and Computing, 2004.

[15] A. Ghasemi and E. S. Sousa, "Collaborative spectrum sensing for opportunistic access in fading environments," in Proceedings of the 1st IEEE International Symposium on New Frontiers in Dynamic Spectrum Access Networks (DySPAN '05), pp. 131136, November 2005.

[16] D. Cabric, A. Tkachenko, and R. Brodersen, "Experimental study of spectrum sensing based on energy detection and network cooperation," in Proceedings of the 1st ACM International Workshop on Technology and Policy for Accessing Spectrum (TAPAS '06), 2006.

[17] T. Ohno, H. Murata, K. Yamamoto, and S. Yoshida, "Field trial of cooperative sensing technique with energy detection," in Proceedings of the 20th IEEE International Symposium on Personal, Indoor and Mobile Radio Communications, pp. 29262929, 2009.

[18] J. Wu, T. Luo, J. Li, and G. Yue, "A cooperative doublethreshold energy detection algorithm in cognitive radio systems," in Proceedings of the 5th International Conference on Wireless Communications, Networking and Mobile Computing (WiCOM '09), pp. 1-4, September 2009.

[19] L. Cheng, B. Henty, D. Stancil, F. Bai, and P. Mudalige, "Mobile vehicle-to-vehicle narrow-band channel measurement and characterization of the $5.9 \mathrm{GHz}$ dedicated short range communication (DSRC) frequency band," IEEE Journal on Selected Areas in Communications, vol. 25, pp. 1501-1516, 2007.

[20] P. M. Shankar, "A compound scattering pdf for the ultrasonic echo envelope and its relationship to $K$ and Nakagami distributions," IEEE Transactions on Ultrasonics, Ferroelectrics, and Frequency Control, vol. 50, no. 3, pp. 339-343, 2003.

[21] L. G. Stuber, Principles of Mobile Communication, Kluwer Academic Publishers, Boston, Mass, USA, 2001.

[22] J. D. Lewinskey, "Non stationary probabilistic target and cluttering scattering models," IEEE Transactions on Aerospace and Electronic Systems, vol. 31, pp. 490-498, 1983.

[23] H. Rasheed, N. Rajatheva, and F. Haroon, "Spectrum sensing with energy detection under shadow-fading condition," in Proceedings of the 5th IEEE International Symposium on Wireless Pervasive Computing (ISWPC '10), pp. 104-109, May 2010.

[24] S. P. Herath and N. Rajatheva, "Analysis of equal gain combining in energy detection for cognitive radio over Nakagami channels," in Proceedings of the IEEE Global Telecommunications Conference (GLOBECOM '08), pp. 1-5, November 2008. 

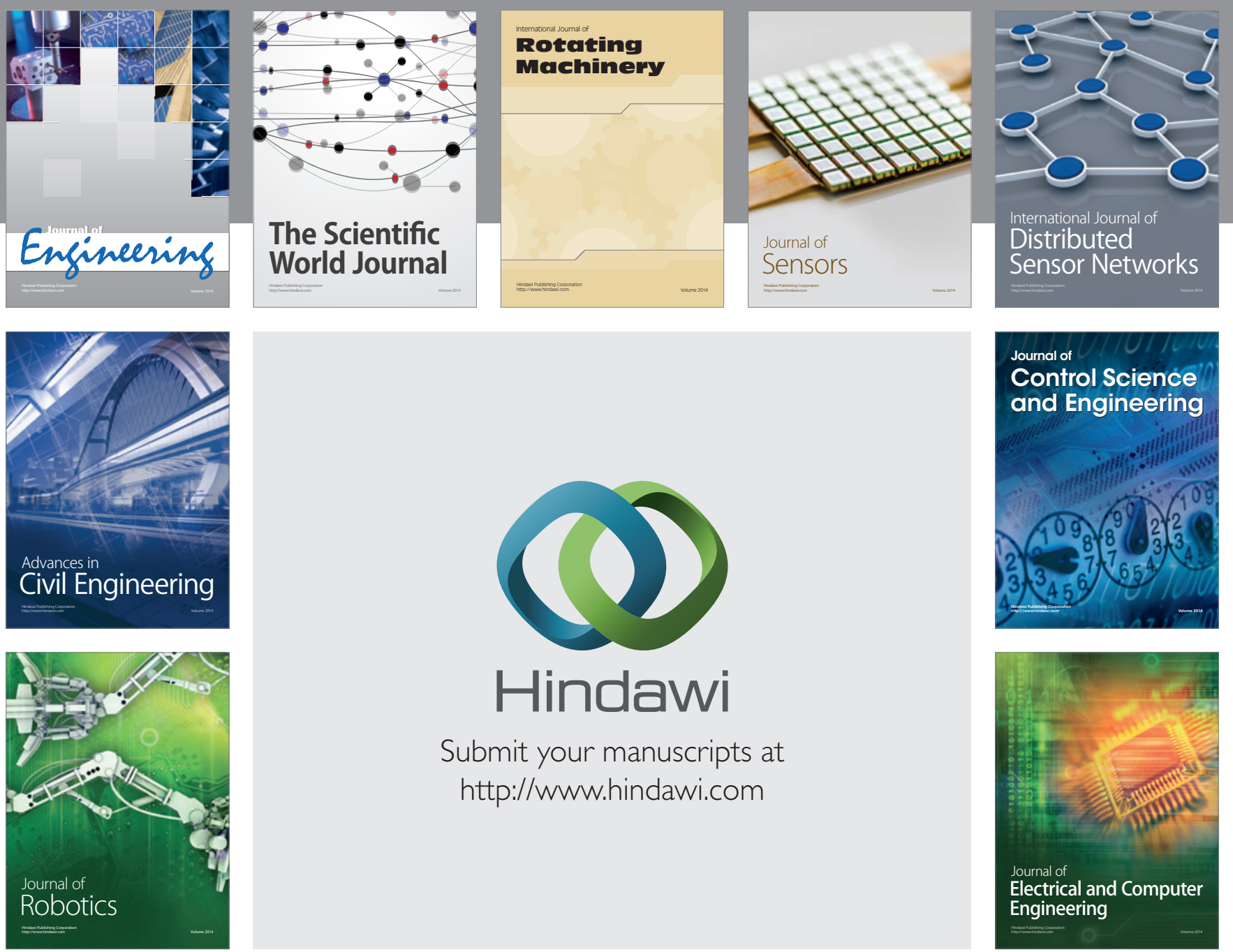

Submit your manuscripts at

http://www.hindawi.com
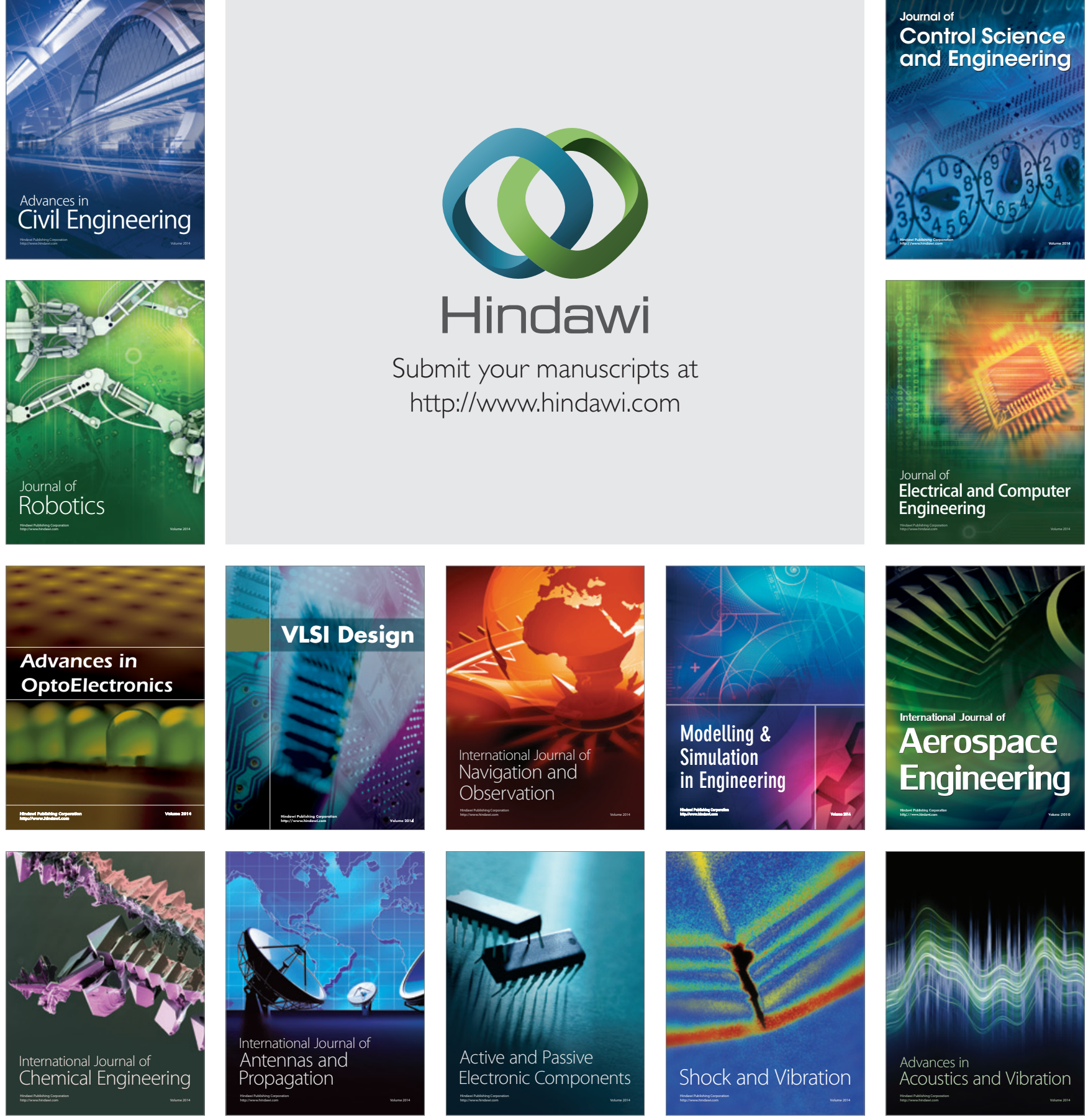